\title{
Pilot Study to Assess Outcomes of a Drug Allergy Clarification Service on a General Medicine Floor at a Local Community Hospital
}

Crystal M. Deas, PharmD, BCPS ; C. Whitney White, PharmD, BCPS ${ }^{2}$

${ }^{1}$ Samford University, McWhorter School of Pharmacy, Clinical Pharmacist, Cooper Green Mercy Health Services, Birmingham, AL

${ }^{2}$ University of Mississippi School of Pharmacy, University of Mississippi Medical Center, Jackson, MS

\begin{abstract}
Purpose: Drug allergy documentation in the patient medical record varies in level of detail, and drug intolerances are often inappropriately documented as an allergy in the medical record. A pilot study was conducted to determine the impact of a pharmacyled drug allergy clarification service.

Methods: The pilot quality improvement service was implemented in Fall 2016. General medicine patients were identified through daily census reporting and the electronic medical record (EMR) was reviewed within 72 hours of admission for documented drug allergies and/or intolerances. Patients were interviewed by a clinical pharmacist or a fourth year pharmacy student to determine a complete drug allergy and intolerance history.

Results: A total of 55 patients were interviewed and received the pilot service. A drug allergy/intolerance was documented in EMR for $54.5 \%(n=30)$ of patients interviewed. Of those 30 patients, $96.6 \%(n=29)$ were noted to have at least one discrepancy between EMR documentation and patient interview. The primary discrepancy noted was drug allergies or intolerances documented in the EMR without a description of the reaction.

Conclusion: A pharmacy-led drug allergy clarification service was effective in identifying and clarifying EMR documentation of patients' drug allergies and intolerances. Patients with incorrect or incomplete allergy documentation may receive alternative therapy, which could increase costs and lead to unwanted adverse effects or less effective treatment. As a result of the pilot study, the program has remained in effect and is being expanded to other units within the institution.
\end{abstract}

Key Words: drug allergy, adverse effect, pharmacy service, electronic health record

\section{Purpose}

The true incidence of drug allergy/hypersensitivity reactions is unknown. In general, drug allergy documentation in the patient medical record varies in level of detail, from no description of the allergic event to single word descriptors (e.g. "rash") to thorough explanation (e.g. "itchy rash over trunk"). ${ }^{1}$ Additionally, drug intolerances or side effects are also often inappropriately documented as an allergy in the patient's medical record. ${ }^{2-8}$ More accurate and comprehensive documentation of allergies improves patient care, as the provider can use the detail to determine if a medication can be safely administered to the patient. Patients with incorrect allergy documentation in the medical record may receive alternative therapy which could be less effective, lead to higher healthcare costs due to prolonged length of stay and/or drug costs, expose patients to unexpected adverse drug effects, and place limitations on future available therapeutic options if the disease or condition recurs. ${ }^{2}$

Corresponding author: Crystal M Deas, PharmD, BCPS

Assistant Professor of Pharmacy Practice

Samford University, McWhorter School of Pharmacy

800 Lakeshore Drive, Birmingham, AL 35229

Phone: 205-726-4079; Fax: 205-726-2669

Email: cdeas@samford.edu
St. Vincent's Birmingham (SVB) is a community hospital located in suburban Birmingham, Alabama. In partnership with Samford University's McWhorter School of Pharmacy, pharmacy faculty serve as affiliate clinical pharmacists and provide clinical services on the general medicine floor at SVB. In the course of the pharmacists' usual practice, the pharmacy faculty will review patients' medical records to conduct comprehensive medication and allergy assessments and provide recommendations to providers. Additionally, the faculty will conduct interviews of the patient and/or caregiver when needed to gather pertinent information related to medications, disease states, or allergies, which supports completion of a more accurate and comprehensive assessment for pharmacy recommendations. Fourth-year pharmacy students who are completing an advanced practice pharmacy experience (APPE) rotation at the facility are also assigned to pharmacy faculty as a part of the faculty member's University obligations. As a part of the general medicine APPE, these students assist the pharmacist with medication and allergy reviews using the patient's medical record, and the students conduct patient/caregiver interviews for the above outlined purpose, when indicated.

Throughout the pharmacists' usual practice on the general medicine floor, errors and omissions in patients' drug allergy and intolerance documentation within the medical record have 
been noted. Some examples of these errors/omissions include: allergies without any listed detail on the type or severity of reaction (allergy description: " $n / a$ ", without detail); documentation with a one-word description of allergy with limited detail (e.g. penicillin - rash vs. penicillin - rash and hypotension); drug intolerances, sensitivities, or expected drug effects documented as a drug allergy (e.g. lorazepam documented as severe allergy; reaction description: drowsiness); and documented drug allergy(ies) in the medical record that the patient does not endorse on interview. When discrepancies have been noted, the pharmacist or APPE student would communicate the information to the provider via faceto-face or written medical record encounters. As a result of this anecdotal experience, this pilot project was developed with the intent to implement a quality improvement service to uniformly clarify patients' allergy history information for a cohort of patients admitted on the general medicine floor. Any identified discrepancies between the medical record and patient interview were communicated to providers and the medical record was updated. The purpose of the pilot was to determine if the service should be continued on the floor and expanded to other areas of the institution.

\section{Methods}

\section{Study design}

A pilot drug allergy clarification (DAC) quality improvement service was implemented during the fall of 2016. The service was conducted by clinical pharmacy faculty and fourth year APPE students. Prior to service implementation, drug allergy clarifications were not routinely conducted within the facility and a standardized format was not utilized for clarification interviews. A retrospective chart review was conducted of patients receiving the DAC service during the initial four weeks of the pilot. Adult patients admitted to the general medicine floor were identified through daily census reporting within the electronic medical record (EMR) and were automatically eligible to receive the pilot service. Patients who were nonverbal, non-English speaking, diagnosed with dementia, or classified as a poor historian in the medical record were excluded from receiving the intervention. This project received exempt status by the St. Vincent's - Birmingham Institutional Review Board.

\section{DAC service}

The DAC service intervention was conducted by clinical pharmacy faculty and APPE students during the pilot [Figure 1]. Prior to participation in the DAC service, APPE students were required to complete a continuing education module on drug allergy clarification, available via Pharmacist's Letter ${ }^{\circ}$, with a requirement of earning passing score of $>80 \%{ }^{1}$. The students participated in role-play exercises with clinical faculty for skillbuilding and observed clinical faculty while conducting the service on an eligible patient. Additionally, faculty observed students as they completed a minimum of two DAC interventions and provided formative feedback and opportunities for remediation of skills as needed. A standardized allergy clarification questionnaire was utilized throughout the DAC service to ensure consistency among clinician interviews [Appendix A].

Within 72 hours of admission, patients' EMRs were reviewed for documented drug allergies and/or intolerances. Additionally during this timeframe, patients were interviewed by a clinical pharmacist or an APPE student to determine a complete drug allergy and intolerance history along with a description of the allergic reaction(s) or medication intolerance(s) - as appropriate. Following the interview, data from the EMR and patient interview were evaluated to determine if any of the following discrepancies were present: (type A) drug allergy or intolerance documented in the medical record without description of reaction; (type B) drug intolerance/sensitivity or known adverse effect of therapy documented in the medical record as an allergy; (type C) drug allergy documented in medical record that patient does not endorse on interview; or (type D) undocumented allergy or drug intolerance discovered upon patient interview. Discrepancies identified were communicated to the hospitalist via verbal communication, when feasible, and medical record documentation. Additionally, patients received education on characteristics of drug allergies and drug intolerances at the conclusion of the patient interview along with tailored education on their specific drug allergy or intolerance, as necessary.

\section{Outcome measures}

The primary outcome measures were the amount of patients with a confirmed true drug allergy upon interview and the total of the following discrepancies: drug allergy or intolerance documented in the medical record without description of reaction; drug intolerance/sensitivity or known adverse effect of therapy errantly documented in the medical record as an allergy; and undocumented allergy discovered upon patient interview. The rate of complete descriptions of the allergic reaction(s) or intolerance(s) among EMR documentation of patients with confirmed true drug allergy versus drug intolerance was evaluated as a secondary outcome.

Drug allergy in this study was defined as symptoms of a type I immediate immunologic reaction, such as hives, itching, angioedema, acute asthma, and anaphylaxis. Drug intolerance was defined in this study as a sensitivity or adverse effect of therapy without an implicated immunologic reaction.

\section{Statistical methods}

Descriptive statistics were used to analyze the primary outcomes. Fisher's exact test was utilized to evaluate the secondary outcome. An alpha value of $p<0.05$ was considered significant. Statistical analyses were conducted using SPSS (IBM Corp. Released 2015. IBM SPSS Statistics for Windows, Version 23.0. Armonk, NY: IBM Corp). 


\section{Results}

During the initial four weeks of the pilot, a total of 55 patients were interviewed and received the pilot DAC service. The mean patient age was 57.1 years, and the majority of patients were female (57.2\%). A drug allergy or intolerance was documented in the medical record for $54.5 \%(n=30)$ of patients interviewed, with a total of 44 discrete drug allergies documented within the EMRs of all patients interviewed. Of the documented drug allergies, true drug allergy was confirmed in the majority of cases $(81.8 \%, \mathrm{n}=36)$.

Of the 30 patients with a documented allergy or intolerance, $96.6 \%(n=29)$ patients were noted to have at least one discrepancy between EMR documentation and patient interview. A total of 45 discrete discrepancies between EMR documentation and patient interview reports were noted. Descriptions of identified discrepancies are provided in Figure 2. It is important to note that of the three type $D$ discrepancies observed, one of which was classified as a true drug allergy discovered upon interview in a patient with "no known drug allergies" documented within the EMR.

The most common discrepancy identified was a drug allergy or intolerance documented in the EMR without a description of the reaction (75.6\%). Upon interview, the most commonly reported reactions to drug therapy were hives/rash $(n=15)$, anaphylaxis/angioedema $(n=6)$, and hallucinations $(n=4)$. A comparison of EMR documentation in confirmed cases of true drug allergy $(n=36)$ versus confirmed drug intolerance $(n=8)$ indicated there was no significant difference in the completeness of EMR descriptions of the reaction(s) or intolerance(s) among groups ( $p=0.5475$, Table 1$)$.

Medications implicated in drug allergy and intolerance discrepancies in this study are described in Table 2. Of the noted discrepancies, antibiotics were the medication class most commonly identified in type $A$ and $D$ discrepancies. Whereas, opioids were more commonly noted in type $B$ discrepancies.

\section{Conclusion}

A pharmacy-led DAC service was effective in identifying and clarifying EMR documentation of drug allergies and intolerances experienced by the majority of patients interviewed during the pilot service. The DAC service was not time-intensive and was seamlessly incorporated into routine clinical care on the floor. Median (IQR) time spent completing the service in those without an identified discrepancy was three minutes (3), whereas the median (IQR) time spent clarifying allergy or intolerance in those with identified discrepancies was 10 minutes (4.5).

The primary discrepancy noted with implementation of the pilot DAC service was drug allergies or intolerances documented in the medical record without a description of the reaction. This could lead to inappropriate prescribing and unnecessary avoidance of beneficial therapies. Additionally, more toxic and costly therapies may be prescribed in cases of type $A$ and type $B$ discrepancies due to incomplete or errant EMR documentation that suggests drug allergy in cases of intolerance. Our study is not the first evaluation to identify the prevalence of incomplete EMR documentation related to reported drug allergies and intolerances. An evaluation conducted by Burrell et al. revealed that EMR records in approximately $47 \%$ of the institutions' patients with a documented drug allergy/intolerance were incomplete and lacked corresponding information describing the drug reaction. Following implementation of a pharmacist-driven protocol to improve documentation of the nature of drug intolerances and reactions, the frequency of complete documentation increased from a rate of $52-62 \%$ to $60-76 \% .{ }^{9}$ Another study, aimed at increasing awareness of the importance of complete chart documentation in postoperative elective surgical patients, evaluated 40 patient charts and found that allergy status documentation was absent in $17.5 \%$ of charts, including $5 \%$ of those with a known drug allergy. After bringing awareness through education, a subsequent chart review showed allergy status documentation improved to $87 \%$ and failure to document a known allergy decreased to $2 \%$. The authors concluded that "collaboration with the pharmacists could also allow a 'best possible medication history to be taken on the day of admission and thus reduce risk to patient safety."10

Other studies have been published examining the impact of pharmacist-led interventions to clarify medication allergies, with the majority focused on clarification of antimicrobial drug allergies. Sigona et al. conducted drug allergy clarification interviews on 32 adults with documented beta-lactam allergy who were receiving non-penicillin antibiotics and noted a discrepancy between the EMR-reported allergy and interview report in $34.4 \%$ of patients. ${ }^{11}$ Similarly, Bouwmeester et al. reported an evaluation of pharmacist interventions to clarify drug allergy reports in a pediatric hospitalized patient population and found that $14 \%$ of documented cases were a true allergy vs. $31 \%$ of cases determined to be incorrectly reported drug allergies. ${ }^{3}$ Our study presents an evaluation of the impact of a comprehensive DAC service to alert providers to discrepancies among EMR documentation and patient interviews for reactions associated with any prescription or non-prescription product.

This study has several limitations. Given the pilot nature of the program, a small, convenience sampling of patients was utilized in a single center, which may limit external validity. Type 1 immunologic reactions were determined to be true drug allergy in this study and delayed type reactions were not considered within the definition. However, it should be noted that there were no reports suggestive of type II and III reactions among patients interviewed in this study. Lastly, this study is an evaluation of a DAC service in an inpatient, general medicine setting. Assessment of the service among various levels of care 
may provide beneficial insight into the effectiveness of the DAC intervention.

More comprehensive documentation of drug allergies and intolerance is warranted within the medical record and should be confirmed at the time of hospital admission. Patients with incorrect or incomplete allergy documentation may receive alternative therapy, which may be more costly, less effective, and associated with unexpected adverse drug effects. As a result of the pilot findings, the program has remained in effect and is being expanded to other units within the facility. Healthcare systems should consider implementation of a similar service for systematic clarification of drug allergies and intolerances to ensure providers are equipped with accurate information to guide therapeutic decision-making.

Conflict of Interest: The authors have nothing to disclose concerning possible financial or personal relationships with commercial entities that may have a direct or indirect interest in the subject matter of this manuscript.

\section{References}

1. Identifying drug allergies and keeping patients safe. Pharmacist's Letter. 2015. Self-study course \#150307. http://therapeuticresearch.com/ce/cecourse.aspx?pc =15-307. Accessed Sept. 1, 2016.

2. Pilzer J, Burke T, Mutnick A. Drug allergy assessment at a university hospital and clinic. Am J Health Syst Pharm. 1996;53:2970-75.

3. Bouwmeester M, Laberge N, Bussiéres J, et al. Program to remove incorrect allergy documentation in pediatrics medical records. Am J Health Syst Pharm. 2001;58:1722-27.

4. Kathawala M. Improving drug allergy documentations using a pharmacist-led campaign. Br J Clin Pharmacol. 2010;2:244-6.

5. Radford A, Undre S, Alkhamesi N, et al. Recording of drug allergies: are we doing enough? J Eval Clin Pract. 2007;13:130-7.

6. Tripp D, Brown G. Pharmacist assessment of drug allergies. Am J Hosp Pharm. 1993;50:95-8.

7. Cantrill J, Cottrell W. Accuracy of drug allergy documentation. Am J Health Syst Pharm. 1997;54:1627-9.

8. Pau A, Morgan J, Terlingo A. Drug allergy documentation by physicians, nurses, and medical students. Am J Hosp Pharm. 1989;46;570-3.

9. Burrell C, Tsourounis C, Quan D, et al. Impact of a pharmacist-driven protocol to improve drug allergy documentation at a university hospital. Hospital Pharmacy. 2013;48(4):302-307. .

10. Thompson A. Improving drug chart documentation in elective surgical patient admissions. BMJ Open Quality. 2014;2:u591.w893.

11. Sigona NS, Steele JM, Miller CD. Impact of a pharmacist-driven beta-lactam allergy interview on inpatient antimicrobial therapy: a pilot project. J Am Pharm Assoc. 2016;56(6):665-669. 
Table 1.

\begin{tabular}{|c|c|c|c|}
\hline \multicolumn{4}{|c|}{ Completeness of EMR descriptions of allergic reaction(s) and drug intolerance } \\
\hline & $\begin{array}{l}\text { Patients with confirmed } \\
\text { drug allergy } \\
\mathrm{n}(\%)\end{array}$ & $\begin{array}{l}\text { Patients with confirmed } \\
\text { drug intolerance }\end{array}$ & p-value \\
\hline Total & 36 & 8 & \\
\hline \multicolumn{4}{|l|}{$\begin{array}{l}\text { Medical record } \\
\text { documentation }\end{array}$} \\
\hline $\begin{array}{l}\text { Complete with } \\
\text { description of } \\
\text { reaction/intolerance }\end{array}$ & $7(19.4)$ & $1(12.5)$ & 0.5475 \\
\hline $\begin{array}{l}\text { Incomplete } \\
\text { description of } \\
\text { reaction/intolerance }\end{array}$ & $29(80.6)$ & $7(87.5)$ & \\
\hline
\end{tabular}

Table 2.

\begin{tabular}{|l|l|l|l|l|l|}
\hline Discrepancies in drug allergies and intolerance by medication class \\
\hline Medication Class & Type $A^{\mathrm{a}}$ & Type $B^{\mathrm{b}}$ & Type $C^{\mathrm{C}}$ & Type $D^{\mathrm{d}}$ & $\begin{array}{l}\text { Number of drug } \\
\text { allergies/intolerances } \\
\text { identified by class }\end{array}$ \\
\hline Antibiotics & 14 & 0 & 1 & 1 & 16 \\
\hline Opioids & 5 & 3 & 0 & 1 & 9 \\
\hline NSAIDs & 2 & 2 & 0 & 0 & 4 \\
\hline Other & 13 & 3 & 0 & 0 & 16 \\
\hline
\end{tabular}

${ }^{a}$ type A: drug allergy or intolerance documented in medical record without description of reaction

${ }^{b}$ type $B$ : drug intolerance/sensitivity or known adverse effect of therapy documented in the medical record as an allergy

'type $C$ : drug allergy documented in medical record that patient does not endorse on interview

${ }^{d}$ type $D$ : undocumented allergy or drug intolerance discovered upon patient interview 
Figure 1. DAC Service Flowchart

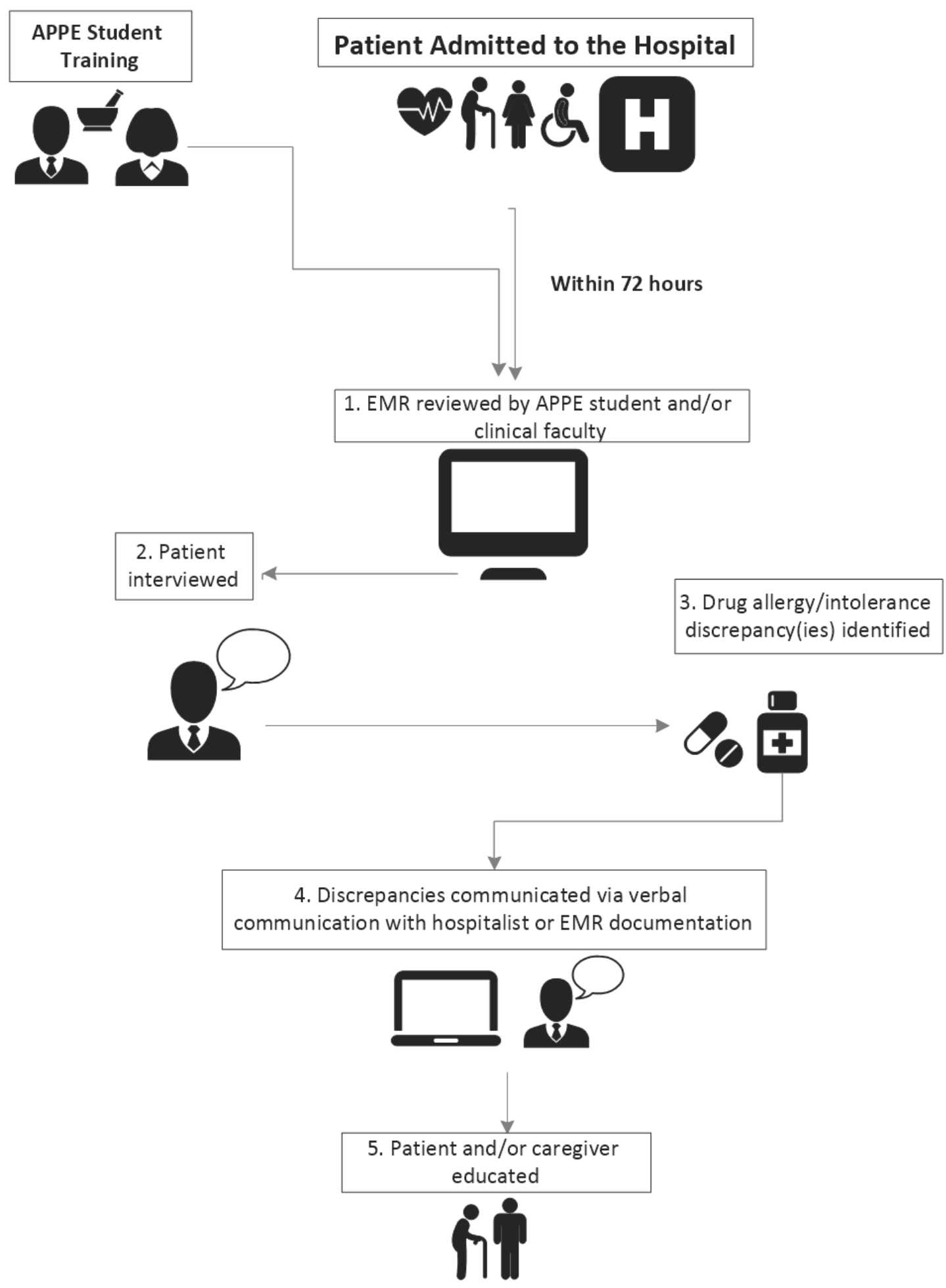


Figure 2.

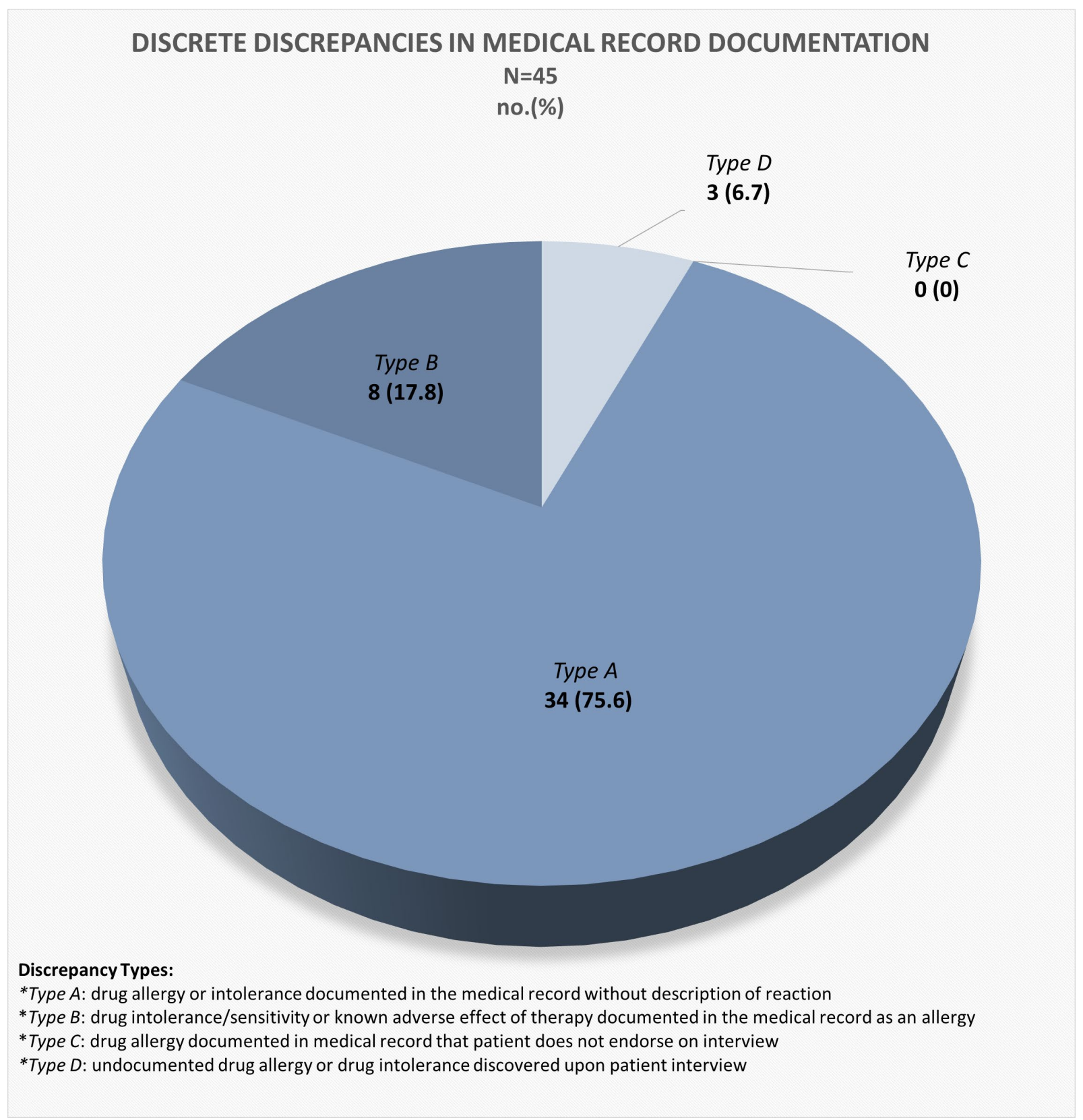


Appendix A.

\section{DAC Standardized Questionnaire}

1. Have you ever taken a medicine that caused a reaction or side effect? If yes, continue to \#2; if no, skip to \#14.

2. What was the medication(s)?

3. Can you tell me why the medication(s) [which caused the reaction/side effect] was prescribed for you?

4. Do you remember the year [or how old you were] when the reaction/side effect to the medication(s) occurred? If yes, ask the specific year or patient's age.

5. Please describe the reaction or side effect. Tell me how the reaction/side effect made you feel.

6. Did you use any prescription medication, overthe-counter medication, or non-medication treatment to stop the reaction/side effect or to make you feel better after the reaction/side effect? If yes, ask the specific medication or non-drug therapy used.

7. After you stopped taking the medication(s) [that may have caused the reaction/side effect], what happened? Did the reaction/side effect resolve?

8. At the time of the reaction/side effect, were you taking any other medication(s) [including over-the-counter and herbal products]?

9. Since taking the [offending] medication(s), have you ever had the same reaction occur but with a different medication(s)?
10. Did you ever take the same medication(s) [that you believe caused the reaction/side effect] again after experiencing the reaction/side effect? If yes, continue to \#11; if no, skip to \#12.

11. What happened when you took the same medication(s) again?

12. Have you ever taken a medication(s) similar to the offending medication after this reaction? Note: interviewer to provide prompts to aid in patient understanding; e.g., in allergy to penicillin VK, the interviewer may ask about use of amoxicillin. If yes, continue to \#13, if no, skip to \#14.

13. Did you have a reaction or side effect to this similar medication? If yes, please describe the reaction.

14. Have you ever experienced any of the following symptoms while taking a prescription or overthe-counter medication:

a. itching, redness, or swelling of the skin;

b. itching, redness or swelling of the eyes;

c. trouble breathing or shortness of breath;

d. swelling of the lips and eye lids;

e. stomach pain

If yes, which symptom(s) occurred and what medication(s) were you taking? 